Puesta en valor de la extensión universitaria: curricularización para una integración real con investigación y docencia

Carla Huarte Bonnet, Andrea Belén Dulbecco, María Soledad Brea, Débora Elizabeth Moricon

Trayectorias Universitarias, 6(11), e040, 2020

ISSN 2469-0090 | https://doi.org/10.24215/24690090e040

https://revistas.unlp.edu.ar/TrayectoriasUniversitarias

Universidad Nacional de La Plata

La Plata | Buenos Aires | Argentina

\title{
Puesta en valor de la extensión universitaria: curricularización para una integración real con investigación y docencia.
}

\section{Enhancement of university extension: curricularization for a real integration with research and teaching}

\section{Carla Huarte Bonnet}

https://orcid.org/0000-0002-0615-2780

carlahb@hotmail.es

CONICET - Instituto de Investigaciones

Bioquímicas de La Plata | Facultad de Ciencias Exactas

| UNLP | Argentina

\section{María Soledad Brea}

https://orcid.org/0000-0002-1438-3984

soledadbrea@yahoo.com.ar

CONICET - Centro de Investigaciones Cardiovasculares

| Facultad de Ciencias Médicas | UNLP | Argentina

\author{
Andrea Belén Dulbecco \\ https://orcid.org/0000-0001-8303-8917 \\ andreadulbecco@gmail.com \\ CONICET - Instituto de Investigaciones \\ Bioquimicas de La Plata | Facultad de Ciencias \\ Médicas |UNLP | Argentina \\ Débora Elizabeth Moriconi
https://orcid.org/0000-0002-6496-4568
debomoriconi@hotmail.com
CONICET - Instituto de Investigaciones
Bioquimicas de La Plata I Facultad de Ciencias
Médicas I UNLP I Argentina
}

RESUMEN

Este trabajo se origina en respuesta a un proceso reflexivo complejo y continuo de nuestras prácticas docentes a lo largo de distintos encuentros, talleres e intercambios de la Especialización en Docencia Universitaria de la Universidad Nacional de La Plata. A lo largo del texto plasmamos nuestras revisiones sobre la fragmentación en la educación y la necesidad de una formación integral, social y colectivamente construida. Consideramos la importancia de la articulación entre docencia, investigación y extensión para los procesos de enseñanza y aprendizaje social, política e históricamente situados, que promuevan un perfil profesional y ciudadano más comprometido con las problemáticas político-sociales, y situamos a la Universidad como actor institucional fundamental en el intercambio permanente con la comunidad. Incluimos también propuestas concretas de curricularización de la extensión como estrategia pedagógica innovadora para implementar en las prácticas docentes universitarias en respuesta a las problematizaciones abordadas.

\begin{abstract}
This work is presented as the result of a complex, continuum and long reflexive process about our teaching practices along different workshops, meetings and collaborative exchanges in our teaching formation journey. The text includes our revisions about educational fragmentation and the importance of an integral, social and collectively constructed professional formation. We consider the importance of the articulation between teaching, research and extension practices for the social, political and historically situated teaching and learning processes, which promote a professional profile more committed to political-social problems, and we place the University as a fundamental institutional actor in the permanent exchange with the community. We also include concrete proposals for curricularisation of extension as an innovative pedagogical strategy to implement in university teaching practices in response to the problems addressed.
\end{abstract}

\section{PALABRAS CLAVE} extensión, curricularización, integralidad, rol social
KEY WORDS

extension, curricularization, integrality, social role 
PRESENTACIÓN DE LA PROBLEMÁTICA: LA FRAGMENTACIÓN EN LA FORMACIÓN UNIVERSITARIA Y LA MARGINACIÓN DE LA EXTENSIÓN DENTRO DE LAS ACTIVIDADES CURRICULARES.

La formación de los universitarios puede estar guiada por dos visiones que implican procesos completamente diferentes: educación como acumulación y educación como construcción social del conocimiento (Rafaghelli, 2013: 24). Teniendo en cuenta esta clasificación, podemos considerar que, de acuerdo a nuestra experiencia como estudiantes y docentes en distintas facultades de la Universidad Nacional de La Plata, en general predomina en este ámbito un enfoque educativo del tipo acumulativo-instructivo, y una pedagogía del tipo liberal y no crítica. Las prácticas y culturas institucionales presentan en general una fragmentación del conocimiento en disciplinas y cátedras, con una deficiencia notable en la cooperación y trabajo transversal entre las mismas, evidenciando un desaprovechamiento en el potencial colectivo de estudiantes y docentes-investigadores en los procesos de enseñanza y aprendizaje. Los perfiles profesionales de las carreras tienden en la práctica a una formación individualista, y desligada del rol social que cada profesión debería contemplar como parte de su accionar. No nos identificamos como actores políticos en una sociedad política, y solo entendemos la responsabilidad social como una actividad caritativa. En este contexto, la extensión universitaria no es prioritaria como actividad formativa, educativa, reflexiva, contemplativa, integrativa. Las actividades de extensión no suelen formar parte de la currícula académica, y la participación de docentes-investigadores es poco valorada y a veces hasta invisibilizada en evaluaciones de concursos docentes, becas, ingresos a carrera y promociones científicas. La vorágine laboral es difícilmente contemplativa con los tiempos invertidos en actividades más ligadas a propósitos sociales. 
La respuesta interna de cada facultad, cátedra o instituto frente a la agenda comunitaria es muy variada, con tradiciones y disputas políticas. Somos nuestra propia limitante en el vínculo con la comunidad.

Por lo tanto, la extensión universitaria tiene por delante desafíos en los planos pedagógico, político, metodológico y epistemológico para, por una parte, superar su desarrollo académico marginal y disociado de la enseñanza y la investigación, su indefinición conceptual y política, y su postergación presupuestal; y por otra contribuir a la transformación de la universidad. Dentro del plano pedagógico, nos parece importante retomar la idea de Cano Menoni (2014: 46) de avanzar en la integración de la extensión a los currículos universitarios y los planes de estudio en todas las áreas de conocimiento, procurando que dicha integración no neutralice su potencial transformador sino que lo vuelque hacia la transformación curricular y pedagógica, incluyendo el nivel de las relaciones educativas, los modelos pedagógicos, así como las concepciones y métodos de evaluación y acreditación de aprendizajes. A su vez, debe procurar su articulación con programas de investigación en diálogo con organizaciones y sujetos populares.

\section{REFLEXIONES HACIA LA INTEGRALIDAD: EDUCACIÓN, INVESTIGACIÓN YEXTENSIÓN.}

Frente a este contexto nos planteamos la importancia de buscar la forma de orientar la agenda académica y científica, manteniendo la excelencia en la producción de conocimientos, hacia la consideración de problemas que afectan a la sociedad. Es aquí donde resulta clave la articulación entre docencia, investigación y extensión. El encuentro entre extensión y enseñanza es fundamental en la detección de demandas y en la transmisión de los valores de la extensión al alumnado en formación. En el encuentro entre enseñanza e investigación se da la identificación de capacidades. La consolidación de la demanda requiere la efectiva articulación entre lo que la sociedad necesita y la investigación, para avanzar en un sentido socialmente útil, y luego poder aplicar los resultados. Pero para lograr esta articulación hay que salir de la Universidad. Y esto significa integrar al interior de la Universidad pero también integrar Universidad y sociedad: estamos hablando de todas las áreas del conocimiento, de la integralidad del conjunto de las tres funciones universitarias con el conjunto de la vida política social.

Haciendo un nexo con toda la sociedad en conjunto, es requisito indispensable que las empresas y el grueso de las organizaciones sociales se vinculen de manera directa con los problemas globales y nacionales; el cambio climático, las guerras, las exigencias democráticas, los movimientos sociales, la pobreza, el desempleo, inestabilidades políticas. Ello exige dejar de pensar en las grandes empresas como agentes externos que poco o nada tienen que ver con los problemas globales, 
nacionales y regionales, sino por el contrario, consiste en exigir que se asuman como parte del complejo social, donde sus acciones repercuten directa o indirectamente. Esta responsabilidad social depende, en gran parte, de la actuación de las universidades como entidad formadora, y es donde cobra sentido una educación orientada a la construcción social. Cuando la mayoría de los empleados estén formados en el tema y se comporten desde dentro como líderes vigilantes de las iniciativas, la responsabilidad social se volverá ya no un lujo, una excepción, sino lo cotidiano, lo que la gente hace. Es ahí donde las universidades encuentran cabida, al introducir estudios sobre el tema, convertir consumidores, empleados, pero más que nada ciudadanos informados, responsables y comprometidos, a su vez, con el desarrollo social (Gasca-Pliego yOlvera-García,2011: 47).

Frente a esta problemática, creemos que un primer abordaje sería impulsar una educación orientada a la construcción social del conocimiento. Es fundamental crear las condiciones para que eso suceda, y es de suma importancia articular una formación integral entre todas las ramas fundamentales de la Universidad. Como sucede con todo cambio profundo en el sistema educativo, el cambio estructural debe ir acompañado de un cambio de concepción: la responsabilidad social no es filantropía, no son buenas intenciones, no son meros principios éticos, no son obras de caridad; lo más cercano a la responsabilidad sería la acción; acción en el entorno, en la sociedad, en la comunidad, dirigida desde las grandes empresas y desde las organizaciones universitarias. Nos parece interesante rescatar la reflexión que Jara Holliday (2018: 236) realiza en cuanto a la educación, la cual debe ser concebida como un proceso humano y no como una actividad aislada, de humanización individual y colectivo dinámico, activo, creador en el que intervienen sujetos y actores específicos (educadores(as) y educandos(as)), que se interrelacionan mutuamente para gestar nuevas capacidades, ideas y procesos. Un proceso históricamente determinado por las condiciones materiales y espirituales de la época y del contexto económico, social, político y cultural concreto de los sujetos que lo llevan a cabo y que constituyen, a su vez, las condiciones sobre las cuales actuar para adaptarse a ellas y mantenerlas o para transformarlas.

Como sucede con todo cambio profundo en el sistema educativo, el cambio estructural debe ir acompañado de un cambio de concepción: la responsabilidad social no es filantropía, no son buenas intenciones, no son meros principios éticos, no son obras de caridad; lo más cercano a la responsabilidad sería la acción; acción en el entorno, en la sociedad, en la comunidad, dirigida desde las grandes empresas y desde las organizaciones universitarias. 
Lograr contextualizar la actividad académica como cultural e históricamente determinada es crucial para potenciar la formación de los universitarios. Los procesos de formación no pueden darse separados y abstraídos de la historia y la cultura. Los fenómenos sociales, psicológicos y culturales deben comprenderse en el contexto de los oficios que cada campo profesional tiene. Consideramos clave empezar a incorporar la participación de los universitarios en la comunidad desde los inicios de su formación, creando instancias transversales en cada materia con la comunidad, para permitir apropiarse de los modos articulados de conocer, de sentir y de actuar. La transversalidad incluye reconocer saberes y conocimientos, abrir la universidad para que no sea autosuficiente y pensar cómo la universidad informa, devuelve y articula con la agenda comunitaria. Creemos que vivenciar la experiencia del contacto con actores directos de las problemáticas sociales es clave para modificar la perspectiva de quien se forma, aunque se dedique luego específicamente a otro campo. En este sentido, el rol de la extensión es clave, ya que apunta al trabajo en conjunto de todos los actores involucrados.

La educación de la ciudadanía debe ser rescatada en toda su magnitud, donde la universidad actúe como modulador clave en los procesos de democratización; ello implica que la universidad debe abrirse hacia la sociedad y para la sociedad, estrechando los lazos que la vinculan, partiendo del diálogo entre todos los actores universitarios (administrativos, docentes, investigadores y alumnado), a través de proyectos que vinculen al alumno con la realidad y le permitan llevar a la acción lo que aprende en las aulas, entendiendo para qué aprende. Las investigaciones deberían vincularnos con los alumnos y responder a necesidades sociales específicas, con investigadores que se permitan interactuar con la realidad que estudian y, a su vez, promuevan la participación de actores ajenos a los espacios universitarios.

\section{DARLE AL VÍNCULO CON LA SOCIEDAD EL ESPACIO QUE MERECE EN LA UNIVERSIDAD: LA CURRICULARIZACIÓN DE LA EXTENSIÓN.}

Para combatir la fragmentación de las tareas universitarias y favorecer el vínculo con la sociedad, la universidad debe comenzar por conjugar nuevas estrategias pedagógicas que promulguen la integración de la responsabilidad social como parte de la educación. Algunas alternativas pueden ser la conformar comunidades de aprendizaje, promover el aprendizaje basado en problemas y proyectos sociales, promover la inter, multi y transdisciplinariedad, revisar continuamente la estructura curricular respecto a los problemas de la agenda de desarrollo local y global, desarrollar actividades pedagógicas de investigación, acción e investigación aplicada en relación con actores no universitarios, conformar equipos multidisciplinarios de investigación, desarrollar líneas de investigación con pertinencia social, promover la responsabilidad social 
desde sus áreas administrativas y de dirección, no solamente desde sus áreas sustantivas, así como procesos democráticos y participativos de toma de decisiones y en la formulación de políticas institucionales de todos los actores internos de la universidad.

En tal sentido, proponemos la generación dentro de todas las carreras de instancias/asignaturas que involucren el estudio de situaciones sociales locales, y el planteamiento de ideas/proyectos para solucionar la problemática planteada, desde la carrera que se esté estudiando. De esta manera se apuntaría a la curricularización de la extensión, donde los estudiantes salen a terreno, aprenden y detectan preguntas junto a los docentes que trabajan en extensión.

Concretamente, imaginamos que una forma de implementar progresivamente estos cambios sería, por un lado, planear, proyectar y ofrecer una materia optativa dentro de las carreras universitarias que tenga como finalidad vincularse con la agenda comunitaria, elegir un potencial problema, problematizarlo, plantear su posible abordaje mediante una construcción interdisciplinar guiada por sus docentes, vinculándose también con actores directa e indirectamente involucrados (grupo social de interés, extensionistas que trabajen en temas o proyectos afines, entes gubernamentales), y planificar su concreción ya sea en lo inmediato o mediato de acuerdo a las posibilidades específicas de cada caso. Asimismo, esta asignatura opcional podría estar disponible para distintas carreras y así permitir un abordaje multidimensional desde cada una de las incumbencias e intereses de los estudiantes.

Concretamente, imaginamos que una forma de implementar progresivamente estos cambios sería, por un lado, planear, proyectar y ofrecer una materia optativa dentro de las carreras universitarias que tenga como finalidad vincularse con la agenda comunitaria, elegir un potencial problema, problematizarlo, plantear su posible abordaje mediante una construcción interdisciplinar guiada por sus docentes, vinculándose también con actores directa e indirectamente involucrados (grupo social de interés, extensionistas que trabajen en temas o proyectos afines, entes gubernamentales), y planificar su concreción ya sea en lo inmediato o mediato de acuerdo a las posibilidades específicas de cada caso.

Por otro lado, siendo que nuestra formación académica incluyó la realización de un trabajo final/tesina de investigación, y que muchas carreras así lo requieren, podría pensarse la implementación de un reglamento para estos trabajos finales que incluya la opción de concretar un plan definido o a definir dentro de un proyecto o programa de extensión. Creemos que esta opción puede solucionar a corto plazo 
algunos inconvenientes frecuentes: el acercamiento de los estudiantes a los proyectos y programas de extensión sería más frecuente y eficaz, mejorando también la divulgación de grupos de trabajo extensionistas formados y en formación; los investigadores-extensionistas y docentes-extensionistas serían los directores y guías de estos trabajos finales y podrían materializar una de las aristas de la labor extensionista en sus curriculum vitae en términos de formación de recursos humanos que hoy se exigen en las presentaciones a becas, ingresos y concursos; los grupos de trabajo contarían con recursos y tiempos más concretos para abordar un problema, y mismo podrían retomarse y utilizar como herramienta planificaciones de la asignatura optativa anteriormente descripta, en casos por ejemplo donde los tiempos de una asignatura no sean suficientes para plantear y concretar un plan de manera inmediata. Sería una opción también pensar el recorrido de manera continua de un estudiante o grupo de estudiantes, en el que trabajen con una problemática de interés durante la asignatura optativa, planteen distintas aristas de trabajo para cada uno de los integrantes del grupo, y en sus respectivos trabajos finales tomen como partida sus construcciones colectivas para la puesta en labor de esas distintas aristas.

También, consideramos que una forma de incluir a la extensión dentro de la currícula de las materias troncales es contando con cargos docentes exclusivos o semiexclusivos a la extensión, de manera que se planteen e implementen proyectos de extensión relacionados con la currícula específica que puedan ser realizados por el conjunto estudiantes-docentes dentro del marco de cada asignatura, y colaborando con la transformación del perfil profesional de los estudiantes (y docentes) hacia el compromiso social y democrático.

Es importante volver a destacar que los cambios deben ser incentivados y respaldados institucional y gubernamentalmente, acompañados de políticas económicas y evaluadoras que permitan solventar la realización de proyectos colectivos e interdisciplinarios, la formación de recursos humanos en extensión, la distribución de los tiempos para investigación, docencia y extensión de manera organizada y equilibrada, y la comunicación abierta y constructiva entre Universidad y sociedad.

Para finalizar, uno de los desafíos que tenemos como docentes es la reflexividad crítica que debemos hacer sobre nuestras prácticas, ya que es la fuente de formación permanente más importante con la que contamos. Todo proceso educativo que busque generar transformaciones y aprendizajes significativos, tendrá necesariamente que considerar, reservar y garantizar espacios para la reflexión, la investigación, la sistematización de experiencias, el encuentro y debate crítico entre educadores/as, como condición esencial de su propuesta pedagógica. Es clave generar estos espacios dentro de la cátedra, del grupo de trabajo, del instituto, de la facultad, de la universidad, y de la sociedad de la que somos parte.

\section{NOTA}

Parte de la producción del presente artículo fue elaborada en el marco del Taller Políticas de articulación, docencia, investigación, extensión y transferencia de la Especialización en Docencia Universitaria, año 2020. 


\section{BIBLIOGRAFIA}

Cano Menoni, José Agustín (2014). “La extensión universitaria en la transformación de la universidad latinoamericana del siglo XXI: disputas y desafíos". CLACSO. Buenos Aires.

Gasca-Pliego, Eduardo y Olvera-García, Julio César (2011). “Construir ciudadanía desde las universidades, responsabilidad social universitaria y desafíos ante el siglo XXI". Convergencia, Revista de Ciencias Sociales, núm. 56. Universidad Autónoma del Estado de México.

Jara Holliday, Oscar (2018). “Aportes de los procesos d Educación Popular a los procesos de cambio" en Educación popular y pedagogías críticas en América Latina y el Caribe: corrientes emancipadoras para la educación pública del Siglo XXI. Ciudad Autónoma de Buenos Aires, CLACSO.

Rafaghelli, Milagros (2013)."La dimensión pedagógica de la extensión”en Integración docencia y extensión. Otra forma de enseñar y de aprender. UNLP. 